\title{
LA POLÍTICA EXTERIOR FEMINISTA (PEF) DE CANADÁ, 2015-2019. EVALUACIÓN Y LECGIONES PARA MÉXICO
}

\author{
CANADA'S FEMINIST FOREIGN POLICY: 2015-2019. \\ ASSESSMENT AND LESSONS FOR MEXICO
}

POLITIQUE ÉTRANGÈRE FÉMINISTE (PEF) DU CANADA:
2015-2019. ÉVALUATION ET LEÇONS POUR LE MEXIQUE

\author{
Óscar Gabriel Mora López \\ Embajada de México en Canadá \\ oscargml@gmail.com
}
María Cristina Oropeza Zorrilla Embajada de México en Canadá cristina.oropeza@gmail.com

\begin{abstract}
Resumen: Tras casi una década de gobiernos conservadores en Canadá, en 2015 regresó al poder el Partido Liberal, encabezado por Justin Trudeau. La victoria alcanzada con un amplio margen de apoyo le permitió implementar una agenda progresista, que contemplaba entre sus prioridades impulsar la igualdad de género de manera transversal, lo que llevó -entre otras cosas- al desarrollo de una política exterior feminista (PEF). El propósito de este artículo es analizar la conceptualización, instrumentación y resultados de esta iniciativa canadiense, a fin de identificar lecciones que puedan ser de utilidad para México, a un año del lanzamiento de su propia PEF.

Palabras clave: política exterior feminista, PEF; negociaciones comerciales; género; desarrollo internacional; política exterior canadiense.
\end{abstract}

Abstract: After almost a decade of conservative governments in Canada, the Liberal Party returned to power in 2015 under the leadership of Justin Trudeau. His resounding victory enabled him to implement a progressive agenda, one that prioritized cross-cutting gender equality, which led to the development of a feminist foreign policy (PEF, by its acronym in Spanish). The purpose 
of this paper is to analyze the conceptualization, implementation and results of this Canadian initiative, in order to identify lessons that might be useful to Mexico, one year after the launch of its own PEF.

Keywords: feminist foreign policy; PEF; trade negotiations; gender; international development; Canadian foreign policy.

\section{Traducción de Fionn Petch, CM Idiomas}

RÉSumÉ: Après près d'une décennie de gouvernements conservateurs au Canada, en 2015, le Parti libéral, dirigé par Justin Trudeau, est revenu au pouvoir. La victoire obtenue avec une large marge de soutien lui a permis de mettre en œuvre un agenda progressiste, qui incluait parmi ses priorités la promotion de l'égalité des sexes de manière transversale, ce qui a conduit -entre autres-au développement d'une politique étrangère féministe (PEF). Le but de cet article est d'analyser la conceptualisation, la mise en œuvre et les résultats de cette initiative canadienne, afin de dégager des leçons qui pourraient être utiles pour le Mexique, un an après le lancement de sa propre PEF.

Mots clés: politique étrangère féministe ; PEF; négociations commerciales ; genre ; développement international ;

Politique étrangère canadienne.

Traducción de Rafael Segovia, CM Idiomas

Fecha de recepción: mayo de 2020

Fecha de aceptación: febrero de 2021 


\section{INTRODUCGIÓN}

\section{$\mathrm{E}$}

n 2015, Justin Trudeau llegó al poder en Canadá con la promesa de generar cambios sustantivos en diversos aspectos de la política canadiense y, en particular, en su relación con el mundo, lo que quedó de manifiesto con su frase "Canadá está de regreso". ${ }^{1}$ Ello generó la expectativa de que el país reposicionaría su tradicional "legado internacionalista liberal de potencia intermedia”, ${ }^{2}$ a partir de la revaloración del multilateralismo; la participación más activa en las Operaciones para el Mantenimiento de la Paz; el lanzamiento de su candidatura para ocupar un asiento no permanente en el Consejo de Seguridad de la ONU (CSONU) para el periodo 2021-2022; la búsqueda de un equilibrio entre el crecimiento económico y el medio ambiente, así como la promoción de la igualdad de género en todos los ámbitos.

Desde el inicio del gobierno, la transversalización de la perspectiva de género fue un imperativo, la paridad en el gabinete obedecía a la lógica irrefutable de que "estamos en 2015" 3 y su plataforma contemplaba la aplicación de una metodología de análisis basada en género (conocida como GBA+) a todas las políticas públicas, a fin de identificar su impacto en la perpetuación de desigualdades sistémicas.

Esta convergencia de prioridades nacionales e internacionales llevó a la adopción de una política exterior feminista (PEF) enmarcada en un renovado debate sobre feminismo que facilitó la elección de Trudeau. ${ }^{4}$

${ }^{1}$ Colin Robertson, “'Canada is Back': Justin Trudeau's Foreign Policy”, Policy Magazine, noviembre-diciembre de 2017.

${ }^{2}$ Laura MacDonald, "La política exterior de Canadá hacia América Latina, de Harper a Trudeau: ¿un regreso al internacionalismo de potencia intermedia?", Revista Mexicana de Política Exterior, sRe, núm. 114, septiembre-diciembre de 2018.

${ }^{3}$ Jennifer Ditchburn, "Because it's 2015: Trudeau forms Canada's $1^{\text {st }}$ gender-balanced cabinet”, CBC News, 6 de noviembre de 2015.

${ }^{4}$ Rebecca Tiessen y Emma Swan, “Canada's Feminist Foreign Policy Promises: An Ambitious Agenda for Gender Equality, Human Rights, Pea- 
No obstante, al ser la PEF un concepto novedoso, para entender las implicaciones del modelo canadiense es necesario delimitar su contenido y conocer hasta qué punto su desarrollo se enmarca en las experiencias de Suecia, Francia $y$, en cierta medida, Noruega (países pioneros en la materia), ya que ello permite comprender su trascendencia como posible referente para México, país que anunció su compromiso de adoptar una PEF durante el $74 .^{\circ}$ periodo de sesiones de la Asamblea General de las Naciones Unidas, en 2019. ${ }^{5}$

\section{¿QUÉ ES LA POLÍtiCA EXTERIOR FEMINISTA, PEF?}

Según Lee, ${ }^{6}$ la PEF es una aproximación política a temas de seguridad y desarrollo internacional que deriva sus raíces del análisis y los principios de la perspectiva de género. Sin embargo, Alwan y Weldon ${ }^{7}$ consideran que es un plan de acción dirigido hacia aquellos que se encuentran fuera de las fronteras nacionales, guiado por un compromiso con la igualdad de género y que busca resolver los problemas del dominio masculino, desigualdad de género y la devaluación y denigración de aquellos que no se ajustan a los estereotipos tradicionales de género. Como se observa, la primera definición se circunscribe al análisis desde la óptica de género de dos temas específicos: seguridad y asistencia, mientras que la se-

ce, and Security", en Norman Hillmer y Philippe Lagassé (comps,), Justin Trudeau and Canadian Foreign Policy Canada and International Affairs, Reino Unido, Palgrave Macmillan, 2018, pp. 187-205.

${ }^{5}$ Secretaría de Relaciones Exteriores, SRE, "Conceptualizando la política exterior feminista: apuntes para México”, Centro de Investigación Internacional, Instituto Matías Romero, abril de 2020.

${ }^{6}$ Danielle Lee, "What is Feminist Foreign Policy? Analysis of Canada's Feminist International Assistance Policy”, Canadá, University of Ottawa, 2018.

${ }^{7}$ Christine Alwan y Laurel Weldon, "What is Feminist Foreign Policy? An Exploratory Evaluation of Foreign Policy in OECD Countries", European Conference on Politics and Gender, Universidad de Lausanne, Suiza, 8-10 de junio de 2017. Purdue University, 2017. 
gunda contempla una mayor interseccionalidad, concepto central en cualquier intervención feminista.

Thompson y Clement han realizado un esfuerzo por destilar los componentes de una PEF ya que, hasta el momento, el uso esporádico de este concepto se ha centrado en fortalecer apoyos para grupos marginados y asignar recursos para buscar la paridad de género; por ello, argumentan que los países que adopten una PEF deben cumplir los siguientes requisitos:

Definir sus interacciones con otros Estados priorizando la igualdad de género y consagrando los derechos humanos de las mujeres y otros grupos tradicionalmente marginados, asignando recursos significativos para lograr esa visión y buscando, a través de su implementación, interrumpir las estructuras de poder patriarcales y dominadas por los hombres en todas sus palancas de influencia (ayuda, comercio, defensa y diplomacia), informados por las voces de activistas feministas, grupos y movimientos. ${ }^{8}$

En ese sentido, la PEF no debe estar limitada a la implementación de mecanismos que fomenten mayor igualdad en la asignación de funciones en la política exterior, o en la acumulación de iniciativas de igualdad de género, sino que es indispensable darle centralidad a los temas de género desde su diseño, a partir de una visión amplia de derechos no limitada a una concepción binaria de mujeres y niñas como únicas destinatarias de esta política, y extenderla a todos los ámbitos de las relaciones internacionales.

Primeras experiencias de Una PEF

Suecia fue el primer país en anunciar la adopción de una PEF en 2014, ante el reconocimiento de que la igualdad de géne-

${ }^{8}$ Lyric Thompson y Rachel Clement, "Defining Feminist Foreign Policy", International Center for Research on Women, 2019. 
ro está intrínsecamente vinculada con la consecución de los objetivos generales de política exterior del país, tales como el desarrollo económico, la prosperidad y la seguridad. Esta PEF está basada en las denominadas " 3 erres" por los términos en inglés correspondientes a derechos, representación (en los ámbitos público y privado) y recursos (distribución equitativa de presupuestos y fondos de asistencia internacional) ${ }^{9}$ A pesar de la innovación que supuso esta iniciativa, fue criticada por diversos sectores de la sociedad sueca por considerar que existía un doble estándar en tanto que no permeaba las políticas migratorias y de exportación de armas, que generan consecuencias diferenciadas por razones de género. ${ }^{10}$ Ello pone de manifiesto el primer reto de una PEF respecto a su integralidad y la necesidad de definir el interés nacional que orienta a toda política exterior, desde una concepción transformativa de feminismo.

En el caso de Francia, su PEF $^{11}$ tiene como objetivos promover el combate a la violencia sexual, la educación de las niñas y mujeres, y el empoderamiento económico de éstas, los cuales se propone alcanzar principalmente a través de la asistencia internacional canalizada a organizaciones civiles en países en desarrollo. Un segundo aspecto de su PEF complementa un cambio de cultura institucional en su Ministerio de Relaciones Exteriores, que debería traducirse en un mayor número de mujeres en puestos decisorios. En tercer lugar, busca aprovechar su posición de liderazgo en foros como el G7 para focalizar la discusión en proyectos que tengan un impacto positivo en la vida de éstas. ${ }^{12}$ No obstante,

${ }^{9}$ Rachel Vogelstein y Alexandra Bro. "Sweden's Feminist Foreign Policy, Long May it Reign." Foreign Policy, 30 de enero de 2019.

${ }^{10}$ Rachel Elizabeth Irwin, "Lessons from Sweden's feminist foreign policy for global health", The Lancet, 9 de febrero de 2019, vol. 393, núm. 10171.

${ }^{11}$ Jean-Yves Le Drian y Marlène Schiappa, “'Feminist Foreign Policy', Ministère de l'Europe et des Affaires étrangères", France Diplomatie: Ministère de l'Europe et des Affaires Étrangères, 2019.

${ }^{12}$ Teresa Wright, "France Takes Torch Passed by Canada, Will Focus on Gender Equality at G7 Summit", National Newswatch, 7 de abril de 2019. 
la principal aportación de Francia es incorporar la responsabilidad y transparencia a su PEF a partir de "objetivos y métricas declarados, con un mandato de evaluación del progreso anual y la publicación de un informe". ${ }^{13}$

Noruega por su parte ha planteado una PEF de facto, "sin etiquetas", 14 a partir de la Resolución $1325^{15}$ del Consejo de Seguridad de la Organización de Naciones Unidas sobre Mujer, Paz y Seguridad (MPs) y su Plan de Acción para los Derechos de las Mujeres y la Igualdad de Género en la política exterior, que establece cinco objetivos: educación equitativa inclusiva; participación equitativa de las mujeres en la vida política; derechos económicos plenos; eliminación de la violencia; salud y derechos sexuales y reproductivos; más que el desarrollo de una política ex profeso, se trata de la extensión hacia la agenda internacional del tradicional activismo del país en la incorporación de la visión de género y del desarrollo de posiciones informadas desde el feminismo.

De las tres experiencias citadas, Suecia representa quizá el ejercicio más acabado, ya que parte de una perspectiva amplia de derechos y acciones en lo público y lo privado, mientras que Noruega y Francia han circunscrito sus acciones, en su mayoría, a ámbitos específicos de seguridad y asistencia, tal como lo refería Lee en su definición. En ese sentido, las lecciones iniciales dejarían entrever que hay por lo menos dos modelos de PEF, dependiendo de su enfoque y alcance. La constante en todos los casos es que ésta necesita un ambiente doméstico receptivo en el que los temas de género se debatan abiertamente y con regularidad. ${ }^{16}$ Además, para ser eficaz, debe ir más allá del enfoque en las mujeres

13 Thompson y Clement, op. cit., p. 4.

14 Torunn L. Tryggestad, "The Feminist Foreign Policy Agenda: Resolution 1325's Legacy", Peace Research Institute Oslo [blog], 26 de octubre de 2018.

15 Naciones Unidas, Consejo de Seguridad, Resolución 1325 del csonu, s/res/1325 (2000), 31 de octubre de 2000, https://www.un.org/ womenwatch/ods/S-RES-1325(2000)-S.pdf

${ }^{16}$ Irwin, art. cit. 
como individuos y concentrarse en desmantelar las causas estructurales de la desigualdad. ${ }^{17}$

El caso de Canadá comparte similitudes con los anteriores modelos en tanto que se ha concentrado en los mismos ámbitos, particularmente asistencia internacional y seguridad. No obstante, ha intentado realizar una aportación adicional en el ámbito comercial, mediante la inclusión del tema en los tratados de libre comercio que ha negociado. Sin embargo, como se explicará con más detalle posteriormente, para algunos estudiosos esto no ha pasado de la retórica, ${ }^{18}$ aunque podría ser un paso en la dirección correcta si se complementa con acciones más ambiciosas que podrían incluir un régimen de solución de diferencias o sanciones.

\section{LA POlítica eXterior feminista de Canadá}

Canadá es considerado un país pionero en la adopción de la igualdad de género como política pública, la cual quedó plasmada como uno de los preceptos fundacionales en su Carta de Derechos y Libertades (1982). Además, este país ha ratificado todos los tratados internacionales para la igualdad de las personas, incluida la Convención sobre la Eliminación de Todas las Formas de Discriminación contra la Mujer en 1981; en 2000 era parte del Consejo de Seguridad de la ONU cuando se adoptó la Resolución 1325, aun si ese ímpetu disminuyó durante el gobierno conservador de Stephen Harper, que empezó en 2006. ${ }^{19}$

17 "Tackling Inequalities in the Global Economy: Making Canada's Foreign Policy Work For Women" Feminist Foreign Policy Series OXFAM Canada.

18 Valerie Hughes, "Gender Chapters in Trade Agreements: Nice Rhetoric or Sound Policy?", Centre for International Governance Innovation, 9 de octubre de 2019.

${ }^{19}$ Rebecca Tiessen y Krystel Carrier, "The Erasure of 'Gender' in Canadian Foreign Policy under the Harper Conservatives: The Significance of the Discursive Shift from 'Gender Equality' to 'Equality between 
Por su parte, Justin Trudeau hizo del feminismo un postulado central de su plataforma política, lo cual fue potenciado por Chrystia Freeland -primera mujer que ostentó el puesto de Ministra de Relaciones Exteriores- y quien enfatizó la importancia de la agenda de igualdad de género y la promoción de los valores canadienses, incluidos el feminismo y los derechos de las mujeres y las niñas. ${ }^{20}$ Ello tuvo como corolario lógico una PEF con el objetivo de "fomentar que las mujeres y las niñas del mundo tengan la misma voz y los mismos derechos, que se beneficien de las mismas oportunidades y que vivan una vida con la misma seguridad"21 a partir de tres vertientes: la negociación de tratados comerciales incluyentes, el lanzamiento de una política feminista de asistencia para el desarrollo y la promoción de la agenda MPS.

\section{NEgOGIAGIONES COMERCIALES}

La economía canadiense depende del acceso al mercado internacional para su bienestar. ${ }^{22}$ Desde la elección de 1988, cuando Brian Mulroney contendió promoviendo el libre comercio como su principal propuesta de campaña, ha habido un consenso sobre la importancia del acceso a los mercados extranjeros para el crecimiento del país. Cada vez más es un imperativo desarrollar estrategias de diversificación, ya que

Women and Men'”, Canadian Foreign Policy Journal, vol. 21, núm. 2, 2015. pp. 95-111.

${ }^{20}$ Stephen Brown y Liam Swiss, "Canada's Feminist International Assistance Policy: Bold Statement or Feminist Fig Leaf?”, en Katherine A.H. Graham y Allan M. Maslove (eds.), How Ottawa Spends, Canadá, Carleton University, 2017, pp. 117-131.

${ }^{21}$ Adam Chapnick, "The origins of Canada's feminist foreign policy", International Journal: Canada's Journal of Global Policy Analysis, 2019, 74 (2), pp. 191-205.

${ }^{22}$ Jean Daudelin y Meredith B. Lilly, "Alivio inmediato, pero un fiasco a largo plazo”, Foreign Affairs Latinoamérica, 2019, vol. 19, núm. 1, http:// revistafal.com/fal-19-1/ 
actualmente el $75 \%$ de su comercio internacional se concentra en Estados Unidos.

En ese contexto, el primer tramo de la administración de Trudeau estuvo marcado por una intensa actividad en el comercio internacional, ya que se concluyó el Acuerdo de Libre Comercio con la Unión Europea (CETA). Ante las presiones estadounidenses, se vieron obligados a renegociar el Tratado de Libre Comercio de América del Norte (TLCAN); renegociaron asimismo el acuerdo bilateral con Chile, participaron en la negociación del Tratado de Integración Progresista de Asociación Transpacífico (TIPAT) y mantuvieron gestiones con otras regiones y bloques comerciales. En todas ellas, Canadá buscó integrar su denominada "agenda progresista”, que incluye temas de género, medio ambiente y comunidades indígenas, con miras a que mayores sectores de la sociedad se beneficiaran del comercio. No obstante, los resultados de ese esfuerzo son variados, particularmente en lo que respecta a la incorporación de la visión de género de manera sustantiva en los textos de los tratados.

El cETA fue el primer acuerdo comercial que logró concretar la administración Trudeau, si bien la mayor parte de las negociaciones tuvieron lugar durante la administración Harper. Este tratado fue descrito por el gobierno liberal como un acuerdo progresista, aun cuando la única mención directa al tema de género se encuentra en el artículo 8.10, relativo a la no discriminación por razones de género en el tratamiento a los inversionistas. Sin embargo, un reporte de la UNCTAD (siglas en inglés de Conferencia de las Naciones Unidas sobre Comercio y Desarrollo) señala que las metas fijadas por este tratado pueden tener un amplio impacto en las mujeres, más allá de su acceso al comercio y oportunidades labores, ya que un análisis interseccional demuestra que existen múltiples avenidas en las que el CETA puede influir la igualdad de género en el comercio. ${ }^{23}$

23 Naciones Unidas, "Gender and Trade: Assessing the Impact of Trade Agreements on Gender Equality: Canada-Eu Comprehensive Economic 
El TIPAT fue visto inicialmente como un mal necesario ya que de no participar en éste, Canadá habría perdería acceso al mercado asiático y enfrentado mayor competencia de los países asiáticos en el mercado estadounidense. Cuando el presidente Trump anunció la salida de EE.UU. del TIPAT, en Canadá se dio por muerto el acuerdo, aunque posteriormente y gracias al liderazgo de países como Japón, el acuerdo transpacífico volvió a ser una posibilidad, por lo que analistas canadienses promovieron permanecer en este nuevo acuerdo, ante evidencia de que Canadá -y México- podrían obtener mayores beneficios sin la presencia estadounidense. ${ }^{24}$ Sin embargo, Canadá no definió su postura claramente hasta muy entrada la renegociación del acuerdo porque no quería contravenir a la administración del presidente Trump. ${ }^{25}$ Es bien sabido que en Vietnam, durante la Cumbre de APEC entre los países que formaban el TIPAT, Trudeau casi descarrila el proceso al no acudir a la reunión de líderes donde se habría llevado a cabo el anuncio de un acuerdo en principio y, posteriormente, condicionó su apoyo a la incorporación de la agenda progresista, arguyendo que "faltaba mucho trabajo por hacer, particularmente la creación de un capítulo de género, cambios a las reglas de origen y otros". ${ }^{26}$ $\mathrm{Al}$ final, en lo que se consideró una victoria pírrica por su limitado alcance, el TPP pasó a ser el тIPAT, el cual en su preámbulo reafirmaba la importancia de la igualdad de género y en

And Trade Agreement", unctad, ILO, un Women, Unión Europea, 2020, https://unctad.org/en/PublicationsLibrary/UNWomen_2020d1_en.pdf

24 Carlo Dade, Dan Ciuriak, Jingliang Xiao y Ali Dadkhah, "The Art of the Trade Deal: Quantifying the Benefits of a TPP without the United States", Canada West Foundation Trade and Investment Centre, junio de 2017, https:/ / cwf.ca/research/publications / the-art-of-the-trade-deal-quantifying-the-benefits-of-a-tpp-without-the-united-states /

25 Rachel Aiello, “Trudeau 'looked flaky' during trade negotiations, 'humiliated' other leaders: former Australian PM", cTV News, 22 de abril de 2020 .

26 John Paul Tasker, "We weren't ready to close the deal: Trudeau defends Canada's actions on TPP”, Canadian Broadcasting Corporation, 11 de noviembre de 2017. 
su artículo 23 contemplaba la cooperación para que las mujeres accedieran y se beneficiaran de las oportunidades creadas por el tratado. ${ }^{27}$

Durante la renegociación del TLCAN, a pesar del ambiente adverso generado por la administración Trump, Canadá trató de insertar su agenda progresista. ${ }^{28} \mathrm{El} 14$ de agosto de 2017, la ministra Chrystia Freeland hizo públicos los seis objetivos ${ }^{29}$ de su país en este proceso, incluyendo la necesidad de contar con un capítulo de "derechos de género". La renegociación fue para Canadá una negociación defensiva, en la que el objetivo principal era mantener intactas algunas secciones del acuerdo original. El resultado, según Daudelin y Lilly, fue un "acuerdo que no se veía mal para Canadá, aunque no reflejaba las metas idealistas originales". ${ }^{30}$ Los objetivos progresistas que se lograron fueron la adopción de reglas laborales más estrictas, que incluyen la no discriminación y la igualdad de las mujeres en los sitios de trabajo. El TMEC incluye disposiciones que buscan incorporar a más mujeres a las cadenas de valor, sin embargo, al no materializarse la inclusión de un capítulo de género, se perdió la oportunidad de imprimir otras consideraciones sustantivas en la materia en el texto del tratado más importante para Canadá. Por el contrario, se perpetuó un modelo tradicional que favorece a empresas e inversionistas, sin reconocer que el comercio afecta de manera diferenciada a hombres y mujeres. ${ }^{31}$

${ }^{27}$ Stephanie Honey, "Will cPтPP Offer Tangible Improvements for Women?", Centre for International Governance Innovation, 6 de abril de 2018.

${ }^{28}$ Salvador Behar Lavalle, "Del TLCAN al T-MEc", Revista Foreign Affairs Latinoamérica ITAM, 2019, http://revistafal.com/fal-19-1/

${ }^{29}$ Gobierno de Canadá, "Address by Foreign Affairs Minister on the modernization of the North American Free Trade Agreement (NAFTA)", 14 de agosto de 2017, https://www.canada.ca/en/global-affairs/news/ 2017/08/address_by_foreignaffairsministeronthemodernizationofthe northame.html

${ }^{30}$ Daudelin y Lilly, art. cit., 2019.

${ }^{31}$ Laura MacDonald y Nadia Ibrahim, "The New NAFTA is a missed opportunity for gender equality", The Monitor, Behind the Numbers, 
Por otra parte, el 5 de febrero de 2019 entró en vigor la versión renovada del Acuerdo de Libre Comercio entre Canadá y Chile (CCFTA). La nueva versión, moderniza el acuerdo original que data de 1997 e incluye un nuevo capítulo de género, que Canadá consideró como "evidencia de que el gobierno lleva la perspectiva feminista a todos los aspectos de su política exterior", ${ }^{32}$ ello aun cuando no se trata de una propuesta innovadora sino que, en gran medida, retoma el texto del acuerdo previo entre Chile y Uruguay. ${ }^{33}$

A diferencia del TLCAN, el CCFTA asume que los impactos del comercio no son neutrales en cuestiones de género y sienta las bases para el desarrollo de indicadores y la implementación de un análisis horizontal de género. De igual manera, en el capítulo respectivo se hace referencia explícita a los compromisos internacionales adquiridos por ambos países en el marco de la Agenda 2030, donde se promueve la igualdad de género y el empoderamiento de las mujeres y las niñas, se traza un marco para que las partes puedan cooperar en asuntos de comercio y género, compromete a las partes a compartir conocimiento y mejores prácticas para fomentar el emprendedurismo entre las mujeres y su mayor participación en la economía internacional. Además, establece un Comité Binacional de Comercio y Género que determinará las actividades apropiadas para el avance de la agenda de género.

A la par, Canadá y el bloque Mercosur iniciaron la negociación de un acuerdo de libre comercio para el que, por primera vez, se realizaría un análisis GBA+ a lo que Jim Carr, Ministro de Diversificación del Comercio Internacional en ese momento, se refirió de la siguiente manera:

http:/ / behindthenumbers.ca/2019/01/23/the-new-nafta-is-a-missed-op portunity-for-gender-equality/

32 Gobierno de Canadá, About the Canada-Chile Free Trade Agreement (CCFTA), op. cit. .

${ }^{33}$ Haifa Bensalem, Gender as Included in Bilateral and Multi-Party Trade and Integration Agreements, Suiza, Cuts International, 2017, p. 19. 
Muchas pequeñas y medianas empresas en Canadá, incluidas las que pertenecen a mujeres y pueblos indígenas, enfrentan desafíos únicos para acceder a oportunidades de exportación. El análisis GBA+ ayudará a garantizar que los acuerdos de libre comercio de Canadá, como el acuerdo Canadá-Mercosur, aborden el desarrollo comercial y económico de una manera que beneficie a más canadienses. ${ }^{34}$

Lo anterior deja de manifiesto que, a diferencia de otros países, Canadá ha hecho un esfuerzo importante por incluir la visión de género en la agenda comercial, y ello debe ser un referente relevante para la creación de una PEF que pretenda reducir las desigualdades a partir del desarrollo económico y el comercio incluyente. Sin embargo, el efecto de incorporar dispositivos aislados en los textos de los tratados será limitado en cuanto a dar visibilidad al tema y no generará el efecto transformativo que se busca con una PEF. Por ello, es necesario un análisis como el que plantea el método GBA+ desde el inicio de cualquier negociación o esbozo de agenda comercial e, incluso, en las labores cotidianas de promoción del comercio e inversiones. Asimismo, resulta evidente que estas negociaciones han resultado más exitosas e integrales que aquellas que se dan en condiciones de mayor simetría con las contrapartes y no así en contextos dispares como el del CPTPP o el TLCAN.

\section{Asistencia Internacional para el Desarrollo}

En junio de 2017, el gobierno canadiense anunció el lanzamiento de la nueva Política Feminista de Asistencia Interna-

34 Gobierno de Canadá, Global Affairs Canada, "Canada releases summary report on first gender-based analysis plus of free trade, Agreement”, 23 de agosto de 2019, https://www.canada.ca/en/global-affairs/ news /2019/08/canada-releases-summary-report-on-first-gender-based-analysis-plus-of-free-trade-agreement.html 
cional $^{35}$ (CFIAP por sus siglas en inglés), que se convertiría en la parte medular de su PEF. Esta nueva política cambia la perspectiva de cooperación hacia un plan que apoya proyectos específicos que impulsen el empoderamiento de las niñas y mujeres en lugares donde son más vulnerables. Marie-Claude Bibeau, entonces ministra de Desarrollo Internacional, señaló que era la "iniciativa más ambiciosa y progresista en la historia de la diplomacia canadiense", con seis ejes de acción transversal:

a) Igualdad de género y empoderamiento de las mujeres y las niñas. Se busca reducir la violencia sexual y de género, y fortalecer a los Estados y organizaciones que promuevan los derechos de la mujer y el desarrollo de sus capacidades.

b) La promoción de la dignidad humana, a través del acceso de asistencia sanitaria, nutricional y educativa, y otorgar asistencia humanitaria en lugares en conflicto basada en las necesidades particulares de las mujeres y niñas.

c) Plan de crecimiento y fortalecimiento de oportunidades económicas para fomentar la independencia económica de las mujeres.

d) Promoción de medidas de mitigación y adaptación del cambio climático que reconozca el liderazgo de las mujeres y les genere oportunidades de participación en proyectos de energías limpias.

e) Apoyo a una gobernabilidad incluyente, la promoción de los derechos humanos, el Estado de derecho y el fortalecimiento de las instituciones, así como una mayor participación política de las mujeres.

f) Fortalecimiento de la paz y la seguridad internacional mediante la participación de las mujeres en temas de consolidación de la paz y los esfuerzos de reconstrucción posconflicto.

35 Gobierno de Canadá, Ministerio de Relaciones Exteriores, Canada's new Feminist International Assistance Policy. Gobierno de Canadá, 2017, https:/ / www.international.gc.ca/world-monde/issues_development-enjeux_deve loppement/priorities-priorites/policy-politique.aspx?lang=eng\#1 
A pesar de los objetivos ambiciosos de esta política y de la existencia de estudios de impacto previos que demuestran que los programas de asistencia focalizados a grupos de mujeres tienen una mayor eficacia en la eliminación de la pobreza, es escasa la información sobre las métricas que se están utilizando para identificar el impacto de los proyectos financiados, particularmente en su comparación con proyectos anteriores. Ello, toda vez que, aunque a principios de 2019 se dieron a conocer los indicadores centrales de desempeño (KPI por sus siglas en inglés) que se emplearían para dar seguimiento a cada eje, hasta inicios del último trimestre de 2020 no se cuenta con el reporte de resultados correspondiente, que debería publicarse durante el invierno. ${ }^{36}$

El gobierno canadiense definió que, a partir del lanzamiento de la CFIAP, $15 \%$ de la asistencia bilateral se etiquetaría específicamente para iniciativas que permitieran avanzar en la igualdad de género y en mejorar la vida de mujeres y niñas y que, a partir de 2021-2022 se asegurarían de que 95\% de las iniciativas que recibieran fondos canadienses a través de asistencia bilateral tuvieran ese objetivo.

No obstante, algunos analistas consideran que al no tenerse una definición clara de género, ni un enfoque interseccional, las acciones sólo se centran en mujeres y niñas, sin reconocer que la desigualdad es una causa subyacente de la pobreza que, para su erradicación, exige la inclusión de hombres, personas LGBTI y otros colectivos, y que el empoderamiento de las mujeres tiene que verse como un objetivo en sí mismo de la política exterior, y no sólo de la política de asistencia. $^{37}$

36 Gobierno de Canadá, "Feminist International Assistance Policy Indicators", 28 de febrero de 2019, https://www.international.gc.ca/worldmonde/issues_development-enjeux_developpement/priorities-priorites / fiap_indicators-indicateurs_paif.aspx?lang=eng\&_ga=2.90388954.135 9037748.1603159364-1517304726.1601066506

37 Corinne L. Mason, "Buzzwords and fuzzwords: flattening intersectionality in Canadian aid”, Canadian Foreign Policy Journal, vol. 25, núm, 2, 2019, pp. 203-219. 
Asimismo, es importante subrayar que el cambio en las prioridades no trajo consigo un incremento en el presupuesto destinado a la asistencia internacional para el desarrollo. Desde los tiempos del gobierno conservador de Stephen Harper, Canadá destina sólo $0.26 \%$ del producto interno bruto (PIB) a la asistencia para el desarrollo internacional, cifra mucho menor a la recomendación de la ocDE de $0.7 \%$, lo que contrasta, por ejemplo, con el presupuesto en defensa que sí se ha incrementado con Trudeau. De igual manera, cabe señalar que una parte importante de la asistencia para el desarrollo que brinda Canadá se dirige a organismos internacionales cuyas prioridades no necesariamente están alineadas con los objetivos planteados en la CFIAP.

Para ilustrar las acciones y tensiones en el marco de la CFIAP, baste referir los anuncios de recursos destinados a apoyar esfuerzos globales contra la covid-19. Por ejemplo, el 20 de marzo se dio a conocer que se canalizarían 50 millones de dólares (MDD) para apoyar a países vulnerables a enfrentar la pandemia y sólo se hace mención de que los vehículos de distribución de los fondos incorporarán "lecciones aprendidas" en previas emergencias sanitarias para garantizar que las necesidades de mujeres y niñas serán tomadas en cuenta. Fue hasta el 22 de junio (tres meses después de declarada la pandemia) que se informó de un programa vinculado a la salud de mujeres y niñas, en el cual se dio a conocer la inversión de 93.7 MDD en apoyo a iniciativas sobre salud sexual y reproductiva, que incluía compromisos adquiridos desde 2018.

Por otro lado, previamente se habían anunciado 79.21 MDD en asistencia para el desarrollo de nuevos proyectos en las Américas enfocados en los derechos sexuales y reproductivos, prevención de la violencia de género, incremento de la participación política de las mujeres, mejor acceso a fuentes de agua y empoderamiento de mujeres agricultoras y 50.35 MDD destinados a Siria, Líbano, Jordania e Iraq para proyectos de asistencia para servicios de salud sexual y reproductiva. 
En general, estas inversiones se perciben como insuficientes y atomizadas, por lo que de no ampliarse los fondos destinados a asistencia internacional y no extender sus principios al grueso de la política exterior del país, como lo ha intentado hacer Suecia, se corre el riesgo de que esta política emblemática canadiense se convierta en una declaratoria de intenciones con la que se busca etiquetar como feministas iniciativas que no lo son, y en realidad utilizan a mujeres y niñas para alcanzar otros objetivos de desarrollo. ${ }^{38}$

\section{Agenda MUJER, PAZ Y SEgURIDAd (MPS)}

En noviembre de 2017, Canadá lanzó el Plan de Acción sobre Mujer, Paz y Seguridad, MPs, ${ }^{39}$ como mecanismo para instrumentar la Resolución 1325 del csonu. También se creó el cargo de embajadora para MPs, el cual anunció el primer ministro en 2019, y sería ocupado por Jacqueline O’Neill quien tendría las tareas siguientes:

Promover la política exterior feminista de Canadá defendiendo sus compromisos prioritarios de mujeres, paz y seguridad en Canadá y en todo el mundo. También trabajará en todos los departamentos federales y con socios para asesorar sobre la implementación del Plan de Acción Nacional de Canadá sobre Mujeres, Paz y Seguridad. Además de asesorar a los ministros en este desafío crítico, la Sra. O’Neill también recomendará acciones para proteger los derechos de las mujeres que enfrentan la inseguridad y la violencia y promover su participación significativa en nuestros esfuerzos de

${ }^{38}$ Stephen Brown y Liam Swiss, "Canada's Feminist International Assistance Policy: Bold Statement or Feminist Fig Leaf?” (cap. 10 ), Katherine A.H. Graham y Allan M. Maslove (comps.), How Ottawa Spends, Carleton University Press, 2017, pp.117-131.

${ }^{39}$ Gobierno de Canadá, "Canada's National Action Plan on Women, Peace and Security”, 10 de junio de 2020. 
desarrollo, humanitarios y de paz y seguridad, alrededor del mundo. ${ }^{40}$

Es decir, con este nombramiento se pretendía una promoción de la PEF a partir de la articulación de los temas de seguridad y desarrollo, y la coordinación horizontal de la estructura gubernamental de Canadá para "acelerar la implementación de la agenda MPs en Canadá y en el mundo". ${ }^{41}$ El objetivo de Canadá era demostrar su liderazgo en iniciativas que permitieran la integración de la agenda MPs en países frágiles o afectados por conflictos. Para conseguir este objetivo, se lanzó la Iniciativa Elsie ${ }^{42}$ como un programa piloto multilateral con el que se busca incrementar la participación de las mujeres en labores de policía o militares dentro de las OMP, a partir de diversos componentes de cooperación que incluyen un fondo global administrado por onu Mujeres, del que Canadá es el principal contribuyente.

Las acciones de Canadá en el rubro MPs son las más integrales, interseccionales y transparentes de su PEF, ya que se vinculan específicamente con los esfuerzos de la CFIAP para la asignación de recursos, se contempla el trabajo de múltiples ministerios (Defensa, Seguridad, Género, Inmigración, etcétera), se reconoce la necesidad de incorporar una vasta gama de actores de la sociedad incluidos hombres, población LGBTI, etcétera, y cuenta con un detallado sistema para reportar anualmente el progreso alcanzado en la consecución de sus objetivos. ${ }^{43}$ Este marco permite realizar los ajustes necesarios para maximizar la eficacia de las intervenciones y recursos destinados a esta agenda.

${ }^{40}$ Gobierno de Canadá, "prime Minister names first Ambassador for Women, Peace and Security", 12 de junio de 2019.

41 "Five Questions on Gender Equality in Foreign Policy: Jacqueline O'Neill', Council of Foreign Relations, 21 de abril de 2020.

${ }^{42}$ Gobierno de Canadá, Elsie Initiative for Women in Peace Operations, 3 de septiembre de 2020.

${ }^{43}$ Gobierno de Canadá, Progress Report for Canada's National Action Plan 2017-2022 for the Implementation of the United Nations Security Council Resolutions on Women, Peace and Security, 10 de junio de 2020. 
Todas las acciones mencionadas representan la materialización del planteamiento del primer ministro Trudeau al señalar que Canadá estaba "de regreso", vinculadas esencialmente a la participación en OMP y a ocupar un asiento no permanente en el CSONU aunque, a la postre, las iniciativas más visibles fracasaron, ya que Canadá tuvo que limitar el alcance de su participación en la Minusma (algo por lo que fue duramente criticado tanto en el país como en la arena internacional) y perdió en la contienda por el csonu. Esta derrota, que aunque puede entenderse como multifactorial, se vinculó ampliamente con los pocos recursos que se destinan a la asistencia internacional (sobre todo en comparación con los otros contendientes, Noruega e Irlanda) y a la falta de una política exterior que carece de objetivos claros, incluso en el ámbito de la igualdad de género. ${ }^{44}$

\section{Evaluaciones PRELIMINARES DE LA PEF CANAdiense}

Como se observa, la PEF de Canadá no ha estado exenta de retos. Ha sido difícil establecer objetivos tangibles que permitan medir el éxito de las iniciativas, por lo que algunos críticos afirman que la PEF es más bien un esfuerzo de mercadotecnia política. En el área comercial, la organización civil internacional Oxfam ha identificado acciones necesarias para alcanzar una PEF más integral, que incluyen procesos de consulta abiertos en el marco de las negociaciones, extender la responsabilidad de las empresas hacia los compromisos con la igualdad de género y la articulación de la política comercial con la dimensión económica de la cFIAP, a partir de proyectos vinculados con las labores de cuidado no remuneradas y los derechos laborales de las mujeres.

${ }^{44}$ Ann Fitz-Gerald y Hugh Segal, "Canada's long-term foreign policy intentions need a clearer narrative", Policy Options, 16 de septiembre de 2020. 
En cuanto a la asistencia internacional, Christoph Zurcher, de la Universidad de Ottawa señala, por ejemplo, que la CFIAP es demasiado abstracta para ser eficaz y que es necesario ponerla en contexto para que no se considere como una imposición de valores canadienses u occidentales, ajenos a otros países. Además, los requisitos administrativos que impone Canadá a las ONG son onerosos, lo que limita la participación de organizaciones pequeñas, y ello se suma a una deficiencia institucional, ya que la burocracia en la cancillería canadiense no ha podido adaptarse con la suficiente rapidez al nuevo esquema de trabajo que exige la CFIAP. ${ }^{45}$ En ese mismo sentido, Tiessen considera que la CFIAP tiene algunos problemas de diseño y, por lo tanto, no ha tenido el impacto esperado. ${ }^{46}$ Entre los defectos que detecta están que Canadá no define su concepto operativo de feminismo y la ambigüedad se refleja en la operación, además de que la cFIAP mantiene una política de apoyo preponderante hacia niñas y mujeres, lo que deja al margen un esfuerzo más amplio de género que incluiría a la comunidad LGTBI.

Asimismo, faltan mecanismos más claros para evaluar el impacto de sus acciones y cómo se entrelazan con sus objetivos amplios de política exterior. Si Canadá quiere mostrar liderazgo global con su enfoque de género, necesita más financiamiento en las áreas de desarrollo y buscar sinergias con los esfuerzos comerciales y de defensa. También debe encontrar una manera de equilibrar sus objetivos declarados de política exterior con intereses comerciales, como la venta de armamento a países que no respetan los derechos de las mujeres de manera sistemática. Durante mucho tiem-

45 Christoph Zuercher, “Canada's Feminist International Assistance: Can Bad Policy Be Well Implemented?”, Centre for International Policy Studies, 13 de septiembre de 2018.

${ }^{46}$ Rebecca Tiessen, "What's New about Canada's Feminist International Assistance Policy: The Problem and Possibilities of 'More of the Same", Canadian Global Affairs Institute, University of Calgary School of Public Policy, vol. 12, núm. 44, diciembre de 2019. 
po, la venta de vehículos blindados a Arabia Saudita ha generado fuertes críticas. ${ }^{47}$

Sin duda, la PEF de Canadá responde a los valores y la imagen que el primer ministro ha impulsado desde que llegó al poder. Si este enfoque ha de echar raíces, es necesario consolidar sus herramientas, enfoque, procedimientos y presupuesto para que no se elimine en una administración posterior con otro corte ideológico o, incluso, ante reveses como lo acontecido con su candidatura al cSONU.

En suma, la PEF canadiense precisa mayor cohesión, interseccionalidad y objetivos claros a largo plazo, en los que la asistencia internacional, el comercio y la agenda de seguridad hagan de la igualdad de género un fin y no un medio. Es todavía una iniciativa perfectible y se espera que el anuncio del nuevo ministro de Relaciones Exteriores, François-Philippe Champagne, ${ }^{48}$ en el Consejo de Relaciones Internacionales de Montreal sobre el trabajo con la sociedad civil para la publicación de un libro blanco en la materia, fortalezca las bases y mecanismos de diseño e implementación de la PEF.

\section{LeCGiones PARA MÉXICO}

En enero de 2021 se cumple un año del lanzamiento de la PEF mexicana, en ocasión de la 31. ${ }^{a}$ Reunión de Embajadores y Cónsules, misma que está sustentada en cinco pilares, de los cuales dos se enfocan propiamente en la acción internacional: hacer transversal en todas las áreas de la política exterior mexicana el enfoque de derechos humanos, la perspectiva de género e interseccionalidad y asegurar que todos los ejes de acción tengan como principio la PEF. Dos más se enfocan en la estructura de la SRE, y aspiran a una cancillería

${ }^{47}$ Steven Chase, "Human rights advocates urge Trudeau to reverse decision on resuming approval of permits for arms exports to Saudi Arabia”, The Globe and Mail, 15 de mayo de 2020.

${ }^{48}$ Gobierno de Canadá, Address by Minister of Foreign Affairs to the Montreal Council on Foreign Relations, 21 de febrero de 2020. 
paritaria y libre de violencia, mientras que el último, que podríamos llamar mixto, se enfoca en visibilizar las aportaciones de las mujeres tanto en el interior como en el marco del desarrollo de la política exterior.

Si bien en la presentación de la PEF y en diversos foros se ha señalado que contará con un documento rector y un manual de principios, éstos aún no han sido publicados, y en el Programa Sectorial de la SRE 2020-2024 (PSRE) se encuentra una referencia a la PEF vinculada exclusivamente al ámbito multilateral, aunque en otras secciones, particularmente las relacionadas con la cooperación internacional, se hace referencia a la incorporación de la visión de género.

En ese contexto, y en espera de que se expanda el marco teórico y se generen términos de referencia de la PEF mexicana, nuestro país podría fortalecer su enfoque al incorporar algunas de las lecciones aprendidas de la PEF canadiense, entre las que destaca una cuestión primordial: que la PEF mexicana se articule de manera integral y no segmentada como ha sido la experiencia canadiense.

En ese sentido, Canadá tiene en realidad tres políticas feministas: la comercial, la de asistencia y la de seguridad y no una sola PEF integral. Es a través de la agenda MPs que se ha tratado de incorporar los tres rubros, sin embargo, aún es un ejercicio en curso.

En el caso de México, es deseable que el abordaje no sea la promoción de muchas políticas con visión de género, sino un cuerpo congruente que regule todas sus interacciones internacionales. De hecho, al vincular la PEF sólo al ámbito multilateral como sucede en el PSRE, dejaría fuera, por ejemplo, las relaciones bilaterales más trascendentes del país, con sus vecinos del norte y sur, y sólo terminaría incorporando una visión de género en la medida que avancen o no programas de cooperación internacional con dichos países.

Ligado a lo anterior, es necesario definir el interés nacional que se persigue con una PEF, ya que ello permite establecer objetivos, diseñar acciones congruentes y parámetros de medición y rendición de cuentas eficaces. En el caso de Ca- 
nadá, los objetivos planteados tienen que ver con la misma segmentación de sus tres políticas y han derivado en críticas sobre la falta de resultados. Sin embargo, puede establecerse con claridad que, en gran medida, el interés nacional que guía su política exterior es la promoción de sus valores, ya que por construcción identitaria Canadá depende del reconocimiento de esos valores para afirmar su ámbito de influencia como potencia media, por lo que al declarar al feminismo como parte de aquéllos, se ha definido un hilo conductor de su PEF.

En lo que respecta a México, ese interés nacional podría ligarse a la soberanía y el liderazgo en el Sur global, por lo que su PEF tendría que abordar, por ejemplo, temas de política migratoria y poblaciones indígenas, algo que hasta el momento Canadá ha incorporado como materias subsidiarias de las agendas comercial y MPs.

La política comercial encauzada adecuadamente es una herramienta importante para la promoción del desarrollo y la igualdad, y es una parte muy visible de las relaciones internacionales, razón por la que Canadá ha insistido en incluir la visión de género en sus negociaciones comerciales y, con ello, favorecer el empoderamiento económico de las mujeres y grupos tradicionalmente desfavorecidos. Hasta el momento ese tema no ha sido parte del diseño de la PEF mexicana, lo cual puede obedecer a que institucionalmente las negociaciones comerciales no competen de manera directa a la SRE, sino a la Secretaría de Economía. No obstante, se trata de un tema trascendente de las relaciones internacionales que puede fortalecerse, por lo menos por vía de la promoción comercial que realizan embajadas y consulados, aprovechando por ejemplo las menciones y modelos que a instancias de Canadá se incorporaron en el TMEG y el TIPAT.

En cuanto a la agenda MPs, en el documento de posición para el 75. ${ }^{\circ}$ periodo ordinario de sesiones de la Asamblea General de la onu, ${ }^{49}$ México se compromete a publicar su pri-

${ }^{49}$ Gobierno de México, Documento de posición de México en el $75 .^{\circ}$ periodo ordinario de sesiones de la Asamblea General de la Organización 
mer Plan Nacional de Acción. En ese rubro, Canadá tiene un liderazgo indiscutible, al haber publicado su plan desde 2017, con diversos mecanismos de seguimiento y medición, así como la canalización de recursos vía la CFIAP o iniciativas como Elsie. Sin embargo, ha enfrentado críticas respecto a la desconexión del Plan con la situación doméstica, luego de que el informe de la comisión que realizó la Investigación Nacional sobre las Niñas y Mujeres Indígenas Desaparecidas y Asesinadas concluyera que en el país ha habido actos de genocidio en contra de este segmento de la población. En el caso de México, deberían incluirse vínculos con políticas nacionales, a fin de no restarle credibilidad a la PEF y generar un cambio en nuestro país en torno al tema de seguridad.

En ese sentido, como se señaló previamente, en los países donde se ha adoptado una PEF, ello ha sido resultado natural de un debate abierto y constante sobre la igualdad de género y el feminismo, por lo que la PEF debe nutrirse de los desarrollos domésticos en este ámbito, lo que puede resultar complejo si se observa la respuesta a las manifestaciones y señalamientos de colectivos feministas acontecidos en 2020. Uno de los grandes aciertos de Canadá y su principal motor ha sido la visión feminista del primer ministro, ya que ello ha dado la visibilidad necesaria para que todo el gabinete de una u otra forma esté invertido en hacer de la PEF una iniciativa exitosa. En el caso de México, resultaría deseable un ímpetu similar, a fin de asegurar la congruencia y cohesión de la PEF, además de la posibilidad de destinarle los recursos suficientes para su implementación, especialmente en tiempos de austeridad en la gestión gubernamental.

$\mathrm{Al}$ respecto, México debe revisar la programación y destino de sus fondos de cooperación a fin de identificar si se encuentran alineados con la PEF, ya que hasta octubre de 2020 en las acciones y programas de la Agencia Mexicana de Coo-

de las Naciones Unidas, https:/ /www.gob.mx/sre/documentos/documen to-de-posicion-de-mexico-en-el-75-periodo-ordinario-de-sesiones-de-laasamblea-general-de-la-organizacion-de-las-naciones-unidas?idiom=es 
peración Internacional para el Desarrollo (Amexcid) no se identifican acciones específicas destinadas a promover la trasversalización de la igualdad de género ni la aplicación de una visión feminista y de derechos humanos a los proyectos autorizados. Nuevamente en referencia a la experiencia canadiense, es necesario que las intervenciones no sean aisladas ni tengan como población objetivo sólo a mujeres y niñas, sino que persigan un objetivo claro de igualdad, evitando que los esfuerzos resulten atomizados e insuficientes. Para ello es necesario que, de manera gradual, se aplique una evaluación de género como la GBA+ a todas las adjudicaciones de proyectos derivados de fondos conjuntos o específicos, así como a convocatorias, programas y, en general, a todas las acciones de cooperación bilateral o triangular.

\section{Bibliografía}

AiELlo, Rachel, “Trudeau 'looked flaky' during trade negotiations, 'humiliated' other leaders: former Australian PM", cTV News, 22 de abril de 2020, https://www.ctvnews.ca/politics/trudeaulooked-flaky-during-trade-negotiations-humiliated-other-lead ers-former-australian-pm-1.4907338

Alwan, Christine y Laurel Weldon, "What is Feminist Foreign Policy? An Exploratory Evaluation of Foreign Policy in OECD Countries", European Conference on Politics and Gender, Universidad de Lausanne, Suiza, 8-10 de junio de 2017.

Behar Lavalle, Salvador, "Del tlcan al t-Mec", Foreign Affairs Latinoamérica, ITAM, 2019, vol. 19, núm. 1, http://revistafal.com/ fal-19-1/

Bensalem, Haifa, Gender as Included in Bilateral and Multi-Party Trade and Integration Agreements, Suiza, cuts International, 2017, p. 19, http://www.cuts-geneva.org/pdf/STUDY\%20-\%20Gen der\%20and\%20Trade.pdf

Brown, Stephen y Liam Swiss, "Canada's Feminist International Assistance Policy: Bold Statement or Feminist Fig Leaf?”, en 
Katherine A.H. Graham y Allan M. Maslove (eds.), How Ottawa Spends, Canadá, Carleton University, 2017, pp. 117-131.

Chase, Steven, "Human rights advocates urge Trudeau to reverse decision on resuming approval of permits for arms exports to Saudi Arabia", The Globe and Mail, 15 de mayo de 2020, https:/ / www.theglobeandmail.com/politics/article-human-rights-ad vocates-urge-trudeau-to-reverse-decision-on-resuming/

Chapnick, Adam, "The origins of Canada's feminist foreign policy", International Journal: Canada's Journal of Global Policy Analysis, vol. 74, núm. 2, 2019, pp. 191-205.

Dade, Carlo, Dan Ciuriak, Jingliang Xiao y Ali Dadkhah, "The Art of the Trade Deal: Quantifying the Benefits of a TPP without the United States", Canada West Foundation Trade and Investment Centre, junio de 2017, https://cwf.ca/research/publications/ the-art-of-the-trade-deal-quantifying-the-benefits-of-a-tpp-with out-the-united-states/

Daudelin, Jean y Meredith B. Lilly, "Alivio inmediato, pero un fiasco a largo plazo", Foreign Affairs Latinoamérica, vol. 19, núm. 1, 2019, http://revistafal.com/fal-19-1/

Ditchburn, Jennifer, "Because it's 2015: Trudeau forms Canada's $1^{\text {st }}$ gender-balanced cabinet", https://www.cbc.ca/news/politics/canada-trudeau-liberalgovernment-cabinet-1.3304590

"El COVID19 tendrá un impacto más grande en las mujeres", Global MXNews, 20 de julio de 2020, https:/ / global-mx.news/2020/07/ 20/el-covid19-ha-impactado-mas-a-las-mujeres/

Fitz-Gerald, Ann y Hugh Segal, "Canada's long-term foreign policy intentions need a clearer narrative", Policy Options, 16 de septiembre de 2020, https:/ / policyoptions.irpp.org/magazines/ september-2020/canadas-long-term-foreign-policy-intentionsneed-a-clearer-narrative/

"Five Questions on Gender Equality in Foreign Policy: Jacqueline O’Neill”, 21 de abril de 2020, Council of Foreign Relations, https:/ / www.cfr.org/blog/five-questions-gender-equality-for eign-policy-jacqueline-oneill

Gobierno de Canadá, “Address by Foreign Affairs Minister on the modernization of the North American Free Trade Agreement 
(NAFTA)", 14 de agosto de 2017, https://www.canada.ca/en/ global-affairs/news/2017/08/address_by_foreignaffairsminis teronthemodernizationofthenorthame.html

Gobierno de Canadá, Ministerio de Relaciones Exteriores, Canada's new Feminist International Assistance Policy. Gobierno de Canadá, 2017, https://www.international.gc.ca/world-monde/issues_ development-enjeux_developpement/priorities-priorites/policy-politique.aspx?lang=eng\#1

Gobierno de Canadá, "Feminist International Assistance Policy Indicators", 28 de febrero de 2019, https://www.international.gc. ca/world-monde/issues_development-enjeux_developpe ment/priorities-priorites/fiap_indicators-indicateurs_paif. aspx?lang=eng\&_ga=2.90388954.1359037748.16031593641517304726.1601066506

Gobierno de Canadá, “About the Canada-Chile Free Trade Agree ment (CCFTA)”, 2019, https://www.international.gc.ca/tradecommerce/trade-agreements-accords-commerciaux/agr-acc/ chile-chili/about_a-propos.aspx?lang=eng

Gobierno de Canadá, Prime Minister names first Ambassador for Women, Peace and Security, Ottawa, 12 de junio de 2019, https://pm.gc.ca/en/news/news-releases/2019/06/12/primeminister-names-first-ambassador-women-peace-and-security

Gobierno de Canadá, Progress Report for Canada's National Action Plan 2017-2022 for the Implementation of the United Nations Security Council Resolutions on Women, Peace and Security, 10 de junio de 2020, https:/ / www.international.gc.ca /gac-amc/publications/cnap-pnac/progress_reports-rap ports_etapes-2018-2019.aspx?lang=eng

Gobierno de Canadá, Global Affairs Canada, "Canada releases summary report on first gender-based analysis plus of free trade, Agreement", 23 de agosto de 2019, https://www.canada.ca/ en/global-affairs/news/2019/08/canada-releases-summary-re port-on-first-gender-based-analysis-plus-of-free-trade-agree ment.html

Gobierno de Canadá, "Address by Minister of Foreign Affairs to the Montreal Council on Foreign Relations", 21 de febrero de 2020, https://www.canada.ca/en/global-affairs/news/2020/02/ad 
dress-by-minister-of-foreign-affairs-to-the-montreal-council-onforeign-relations.html

Gobierno de Canadá, "Canada's National Action Plan on Women, Peace and Security", 10 de junio de 2020, https://www.interna tional.gc.ca/world-monde/issues_development-enjeux_devel oppement/gender_equality-egalite_des_genres/cnap_wpspnac_fps.aspx?lang=eng

Gobierno de Canadá, "Elsie Initiative for Women in Peace Operations", 3 de septiembre de 2020, https://www.international. gc.ca/world-monde/issues_development-enjeux_developpe ment/gender_equality-egalite_des_genres/elsie_initiative-ini tiative_elsie.aspx?lang=eng

Gobierno de México, Secretaría de Relaciones Exteriores, SRE, Documento de posición de México en el $75 .^{\circ}$ periodo ordinario de sesiones de la Asamblea General de la Organización de las Naciones Unidas, https://www.gob.mx/sre/documen tos/documento-de-posicion-de-mexico-en-el-75-periodo-ordi nario-de-sesiones-de-la-asamblea-general-de-la-organizacionde-las-naciones-unidas?idiom $=\mathrm{es}$

Honey, Stephanie, "Will CPTPP Offer Tangible Improvements for Women?”, Centre for International Governance Innovation, 6 de abril de 2018, https://www.cigionline.org/articles/willcptpp-offer-tangible-improvements-women

Hughes, Valerie, "Gender Chapters in Trade Agreements: Nice Rhetoric or Sound Policy?”, Centre for International Governance Innovation, 9 de octubre de 2019, https://www.cigion line.org/articles/gender-chapters-trade-agreements-nice-rhe toric-or-sound-policy

IR wiN, Rachel Elizabeth, "Lessons from Sweden's feminist foreign policy for global health", The Lancet, 9 de febrero de 2019, vol. 393, núm. 10171, https://doi.org/10.1016/S01406736(19) 30209-0

Le Drian, Jean-Yves y Marlène Schiappa, “Feminist Foreign Policy', Ministère de l'Europe et des Affaires étrangères", France Diplomatie: Ministère de L'Europe et des Affaires Étrangères, 2019, www.diplomatie.gouv.fr/en/french-foreign-policy/hu 
man-rights/events/article/feminist-foreign-policy-op-ed-byjean-yves-le-drian-and-marlene-schiappa-08-03

LeE, Danielle, "What is Feminist Foreign Policy? Analysis of Canada's Feminist International Assistance Policy”, Canadá, University of Ottawa, 2018, https://ruor.uottawa.ca/bitstream/10 393/37379/1/Lee_Danielle_2018_thesis.pdf

MacDonald, Laura, "La política exterior de Canadá hacia América Latina, de Harper a Trudeau: ¿un regreso al internacionalismo de potencia intermedia?", Revista Mexicana de Política Exterior, sRE, núm. 114, septiembre-diciembre de 2018, https:/ / revistadigital.sre.gob.mx/images/stories/numeros/n114/ macdonald.pdf

MacDonald, Laura y Nadia Ibrahim, "The New NAFTa is a missed opportunity for gender equality", The Monitor, Behind the Numbers, http://behindthenumbers.ca/2019/01/23/the-ne w-nafta-is-a-missed-opportunity-for-gender-equality/

Mason, Corinne L. Mason, "Buzzwords and fuzzwords: flattening intersectionality in Canadian aid", Canadian Foreign Policy Journal, vol. 25, núm. 2, 2019, pp 203-219, https://doi.org/10.108 $0 / 11926422.2019 .1592002$

Naciones Unidas, Consejo de Seguridad, Resolución 1325 del CSONU, S/RES/1325 (2000), 31 de octubre de 2000, https:// www.un.org/womenwatch/ods/S-RES-1325(2000)-S.pdf

Naciones Unidas, "Gender and Trade: Assessing the Impact of Trade Agreements on Gender Equality: Canada-Eu Comprehensive Economic and Trade Agreement", unctad, ILO, UN Women, Unión Europea, 2020, https://unctad.org/en/PublicationsLibrary/UNWomen_2020d1_en.pdf

Robertson, Colin, “'Canada is Back”: Justin Trudeau's Foreign Policy”, Policy Magazine, noviembre-diciembre de 2017, http:/ / www.policymagazine.ca/pdf/29/PolicyMagazineNovember December-2017-Robertson.pdf

Secretaría de Relaciones Exteriores (SRE), "Conceptualizando La Política Exterior Feminista: Apuntes Para México”, Centro de Investigación Internacional, Instituto Matías Romero, abril de 2020, https:/ /www.gob.mx/cms/uploads/attachment/file/ 545369/Nota_6-Poli_tica_exterior_feminista.pdf 
"Tackling Inequalities in the Global Economy: Making Canada's Foreign Policy Work For Women”, Feminist Foreign Policy Series OXFAM Canada.

TAsker, John Paul, "We weren't ready to close the deal: Trudeau defends Canada's actions on TPP", Canadian Broadcasting Corporation, 11 de noviembre de 2017, https://www.cbc.ca/news/ politics/justin-trudeau-tpp-canada-not-ready-apec-1.4398824

Thompson, Lyric y Rachel Clement, "Defining Feminist Foreign Policy", International Center for Research on Women, 2019, https://n2r4h9b5.stackpathcdn.com/wp-content/uploads/ 2019/11/ICRW_DefiningFeministForeignPolicy_Brief_Span ish.pdf

Tiessen, Rebecca, "What's New about Canada's Feminist International Assistance Policy: The Problem and Possibilities of 'More of the Same'", Canadian Global Affairs Institute, University of Calgary School of Public Policy, 2019, vol. 12, núm. 44, diciembre de 2019, www.cgai.ca/whats_new_about_canadas_feminist_ international_assistance_policy_the_problem_and_possibili ties_of_more_of_the_same?utm_campaign=pp\&utm medium $=\mathrm{e}$

Tiessen, Rebecca y Emma Swan, "Canada’s Feminist Foreign Policy Promises: An Ambitious Agenda for Gender Equality, Human Rights, Peace, and Security", Norman Hillmer y Philippe Lagassé (comps.), Justin Trudeau and Canadian Foreign Policy Canada and International Affairs, Reino Unido, Palgrave Macmillan, 2018, pp. 187-205.

Tiessen, Rebecca y Krystel Carrier, "The Erasure of 'Gender' in Canadian Foreign Policy under the Harper Conservatives: The Significance of the Discursive Shift from 'Gender Equality' to 'Equality between Women and Men'”, Canadian Foreign Policy Journal, vol. 21, núm. 2, 2015, pp. 95-111.

Tryggestad, Torunn L., "The Feminist Foreign Policy Agenda: Resolution 1325's Legacy”, Peace Research Institute Oslo [blog], 26 de octubre de 2018, https://blogs.prio.org/2018/10/the-femi nist-foreign-policy-agenda-resolution-1325s-legacy/

Vogelstein, Rachel y Alexandra Bro, "Sweden's Feminist Foreign Policy, Long May It Reign”, Foreign Policy, 30 de enero de 2019, 
www.foreignpolicy.com/2019/01/30/sw eden-feminist-foreig npolicy

Wright, Teresa, "France Takes Torch Passed by Canada, Will Focus on Gender Equality at G7 Summit", National Newswatch, 7 de abril de 2019, www.nationalnewswatch.com/ 2019/04/07/fran ce-takes-torch-passed-by-canada-will-fo cus-on-gender-equalityat-g7-summit-3/\#.XKtjYphKiUk

Zuercher, Christoph, "Canada's Feminist International Assistance: Can Bad Policy be Well Implemented?”, Centre for International Policy Studies, 13 de septiembre de 2018, www.cips-cepi.ca/ 2018/09/13/feminist-international-as sistance-can-bad-policybe-well-implemented-part-1/ 